\title{
ON MONTEL'S THEOREM
}

\section{YOSHIRO KAWAKAMI}

1. In this note we shall prove a theorem which is related to Montel's theorem [1] on bounded regular functions. Let $E$ be a measurable set on the positive $y$-axis in the $z(=x+i y)$-plane, $E(a, b)$ be its part contained in $0 \leqq a \leqq y \leqq b$, and $|E(a, b)|$ be its measure. We define the lower density of $E$ at $y=0$ by

$$
\lambda=\lim _{r \rightarrow 0} \frac{|E(0, r)|}{r} .
$$

Lemma. Let $E$ be a set of positive lower density $\lambda$ at $y=0$. Then $E$ contains a subset $E_{1}$ of the same lower densiry at $y=0$ such that $E_{1} \cup\{0\}$ is a closed set.

Proof. Let $r_{n}=1 / n(n=1,2, \ldots)$. There exists a closed subset $E_{1}\left(r_{n+1}, r_{n}\right)$ of $E\left(r_{n+1}, r_{n}\right)$, such that

$$
\left|E_{1}\left(r_{n+1}, r_{n}\right)\right| \geqslant \delta_{n}\left|E\left(r_{n+1}, r_{n}\right)\right| \quad(n=1,2, \ldots),
$$

with $\delta_{n}=1-\frac{1}{n}$. We put

$$
E_{1}=\sum_{n=1}^{\infty} E_{1}\left(r_{n+1}, r_{n}\right)
$$

Then if $r_{n}<r \leqq r_{n-1}$,

$$
\left|E_{1}(0, r)\right| \geqslant \sum_{i=n}^{\infty}\left|E_{1}\left(r_{i+1}, r_{i}\right)\right| \geqslant \delta_{n}\left|E\left(0, r_{n}\right)\right|,
$$

so that

$$
\frac{\left|E_{1}(0, r)\right|}{r} \geqslant \frac{\left|E_{1}\left(0, r_{n}\right)\right|}{r} \delta_{n} \geqslant \frac{\left|E\left(0, r_{n}\right)\right|}{r_{n}} \cdot \frac{r_{n}}{r_{n-1}} \delta_{n},
$$

whence

$$
\lambda=\lim _{r \rightarrow 0} \frac{|E(0, r)|}{r} \geq \lim _{r \rightarrow 0} \frac{\left|E_{1}(0, r)\right|}{r} \geq \lim _{n \rightarrow \infty} \frac{\left|E\left(0, r_{n}\right)\right|}{r_{n}} \geq \lambda .
$$

\section{Hence}

Received December 7, 1955 ; revised April 12, 1956. 


$$
\lim _{r \rightarrow 0} \frac{\left|E_{1}(0, r)\right|}{r}=\lambda
$$

2. We shall prove the following theorem.

TheOREM. Let $f(z)=f(x+i y)$ be regular and bounded in $x>0$, and continuous at a measurable set $E$ of positive lower density $\lambda$ at $y=0$ on the positive $y$-axis. If $f(z) \rightarrow A$ when $z \rightarrow 0$ along $E$, then $f(z) \rightarrow A$ uniformly when $z \rightarrow 0$ in the domain $|y| \leqq k x$, where $k$ is any positive constant.

Proof. By the lemma we assume that $E \cup\{0\}$ is a closed set. Without loss of generality we may assume that $|f(z)| \leqq 1$ and $A=0$. Let $D_{p}:|z|<\rho$, $x>0$ be the half-disc. Let us denote by $u_{\rho}(z)$ the harmonic measure of $E(0, \rho) \cup\{0\}$ with respect to $D_{\rho}$.

If we take $0<\rho<1$ sufficiently small such that $|f(z)| \leqq \varepsilon$ on $E(0, \rho)$, then, by the maximum principle, we have

$$
\log |f(z)| \leqq u_{p}(z) \cdot \log \varepsilon \text { for } z \in D_{p}
$$

hence

$$
|f(z)| \leqq \varepsilon^{u_{p}(z)} \quad \text { for } \quad z \in D_{p} .
$$

As is well known,

$$
u_{\rho}(z)=\frac{1}{2 \pi} \int_{E(0, \rho)} \frac{\partial}{\partial n} G(i \eta, z) d \eta
$$

where $G_{\rho}(w, z)(w=\xi+i \eta)$ is the Green's function of $D_{\rho}$ with pole at $z=x+i y$. By a simple calculation we have

$$
\left(\frac{\partial G}{\partial n}\right)_{\xi=0}=\frac{2 x\left(\rho^{2}-x^{2}-y^{2}\right)\left(\rho^{2}-y_{i}^{2}\right)}{\left\{x^{2}+(y-\eta)^{2}\right\}\left\{(\rho-y \eta)^{2}+x^{2} \eta^{2}\right\}} .
$$

Hence

$$
\boldsymbol{u}_{\rho}(z)=\frac{1}{2 \pi} \int_{0}^{\rho} \frac{2 x\left(\rho^{2}-x^{2}-y^{2}\right)\left(\rho^{2}-\eta^{2}\right)}{\left\{x^{2}+(y-\eta)^{2}\right\}\left\{\left(\rho^{2}-y \eta\right)^{2}+x^{2} \eta^{2}\right\}} d \mu(\eta)
$$

where

$$
\mu(\eta)=\int_{E(0, r,)} d \eta=|E(0, \eta)| .
$$

If $|z| \leqq \delta \rho, \eta \leqq \delta \rho,(0<\delta<1)$, then

$$
\left(\rho^{2}-x^{2}-y^{2}\right)\left(\rho^{2}-\eta^{2}\right) \geqq \rho^{4} C_{1}, \quad\left(\rho^{2}-y \eta\right)^{2}+x^{2} \eta^{2} \leqq \rho^{4} C_{2},
$$

whence 


$$
u_{p}(z) \geqslant C_{2} \int_{0}^{\delta_{p}} \frac{x}{x^{2}+(y-\eta)^{2}} d \mu(\eta)
$$

where $C_{1}, C_{2}, C_{3}$ are constants, depending on $\delta$ only. Hence if $|y| \leqq k x$, we have

$$
u_{p}(z) \gtrsim C_{3} \int_{0}^{\delta \rho} \frac{x}{x^{2}+(\eta+k x)^{2}} d \mu(\eta) .
$$

By the substitution $y_{1}=x t$, we have

$$
\begin{aligned}
U_{\rho}(z) & \geqslant \frac{C_{3}}{x} \int_{0}^{\delta \rho / x} \frac{1}{1+(t+k)^{2}} d \mu(x t) \\
& =\frac{C_{3}}{x}\left[\frac{\mu(x t)}{1+(t+k)^{2}}\right]_{0}^{\delta \rho / x}+\frac{2 C_{3}}{x} \int_{0}^{\delta_{p} / x} \frac{\mu(x t)(t+k)}{\left\{1+(t+k)^{2}\right\}^{2}} d t \\
& \geqslant 2 C_{3} \int_{0}^{1} \frac{\mu(x t)(t+k)}{\left\{1+(t+k)^{2}\right\}^{2}} d t .
\end{aligned}
$$

Since $\mu(x t) \geqslant \lambda^{\prime} x t$ for some $\lambda^{\prime}$ such that $0<\lambda^{\prime}<\lambda$, we have

$$
u_{\rho}(z) \geq 2 C_{3} \int_{0}^{1} \frac{\lambda^{\prime} t(t+k)}{\left\{1+(t+k)^{2}\right\}^{2}} d t=C,
$$

where $C$ is a constant depending on $k, \hat{o}$, and $\lambda^{\prime}$ only. Hence by (1)

$$
|f(z)| \leqq \varepsilon^{c}, \quad \text { if } \quad|z| \leqq \delta \rho \text { and }|y| \leqq k x,
$$

so that $\lim _{z \rightarrow 0} f(z)=0$ uniformly, when $z \rightarrow 0$ in the domain $|y| \leqq k x$.

Remark. The writer has proved that our theorem holds when $E$ satisfies the condition that $\lambda_{a}$ is positive, where

$$
\lambda_{\alpha}=\lim _{r \rightarrow 0} r^{\alpha-1} \int_{r}^{1} \frac{d \mu(t)}{t^{\alpha}} \quad(\alpha \geq 2) .
$$

However, Professor Ohtsuka kindly informed him that this condition for any $\alpha>1$ is equivalent to the condition that the lower density of $E$ at $y=0$ is positive. ${ }^{1)}$

\section{REFERENCE}

[1] P. Montel, Sur les familles de fonctions analytiques qui admettent des valeurs exceptionelles dans un domaine, Ann. Sci. Ecole Norm. Sup. (3), 23 (1912), pp. 487-535.

Seikei University, Tokyo

1) See the paper after the next. 